\title{
The neutrino event reconstruction in the ICARUS T600 LAr TPC
}

\section{Stefan ${ }^{1}$ on behalf of the ICARUS Collaboration}

Laboratori Nazionali del Gran Sasso dell'INFN,

S.S 17 BIS km. 18.91067010 Assergi L'Aquila, Italy

E-mail: dorota.stefanelngs.infn.it

The Liquid Argon Time Projection Chamber (LArTPC) has excellent imaging capability which provides a unique view of the particle interactions and is, often compared to the old bubble chamber analogue era. Recorded information is so reach in details that special reconstruction algorithms had to be developed and some aspects are still a challenge. In these days the progress becomes fast around the world - full size detectors appear and data is coming. Among the competitors, the ICARUS detector is the biggest one, with the ability to capture full contained neutrino events with all their variety and complexity. In the paper is shortly described the method of the event reconstruction applied for the ICARUS data: the hit reconstruction, the twodimensional event clustering, a three-dimensional track reconstruction approach, and finally stopping particle calorimetric reconstruction.

XV Workshop on Neutrino Telescopes

March 11-15, 2013

Venice, Italy

1

D. Stefan 


\section{Introduction}

Currently the Liquid Argon Time Projection Chamber (LAr-TPC) technology [1] is of great interest due to its potential in investigation of the neutrino properties and rare events such as proton decay or dark matter. The ICARUS detector is the largest liquid Argon (LAr) detector which has ever been built, with mass of 600 ton (T600). The experience gained from the detector construction, operation and data analysis should be further used in the future LAr detectors. The advantage of the LAr TPC is its excellent spatial and calorimetric resolution which makes possible a perfect visualisation of the charged particles tracks. Such a detection technique is often compared to the bubble chamber filled with heavy liquids, but with an electronic readout. In order to take advantage of the modern experimental techniques, the track reconstruction and data analysis should be fully automatized. This requires studying new reconstruction methods and solving many algorithmic problems, which are similar to those from automatic image processing and pattern recognition.

\section{Approach to the event reconstruction}

A charged particle traversing liquid argon produces ionization electrons along its path. Ionization electrons drift in the uniform electric field applied to the LAr active volume toward anode. The anode is composed by three wire planes with different wire orientation; the first two are read-out in induction-mode, the third in collection-mode. The drifting charges produce on each wire plane a two-dimensional projection of the particle track. The combination of these projections drives to a 3D geometrical reconstruction of the event; collection plane signals provide calorimetric information [2]. Excellent granularity of 2D images comes at the price of a complex image recognition problem. Automatic association of the physical objects in complementary wire planes are presently main efforts in the development of reconstruction algorithms.

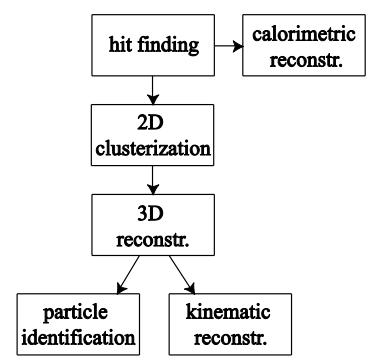

Fig. 1. Scheme of the event reconstructions procedure.

In the event reconstruction two types of objects are distinguished: tracks and cascades. The scheme of the reconstruction procedure is presented on the figure 1. First, the signals (hits) on all three independent wire planes have to be found. Then, only in Collection, the fitting procedure is applied to the signal shape to obtain the charge deposition on wires. At this stage, a rough estimation of the calorimetric measurement can be provided. However, for a full and detailed event kinematical reconstruction, we have to proceed to two further steps, which 
consist in the aggregation of hits in clusters that form 2D structures (tracks and showers) and in the combination of them to finally get the $3 \mathrm{D}$ object reconstruction.

\subsection{Identification of individual signals}

Individual signal identification, so called hit, is the essential base for the spatial and calorimetric measurements. Hits are identified in all wire planes following the algorithm presented in [2]. As a result, hit positions in the drift coordinate are obtained together with the drift time windows, which cover the hit ADC waveform with a margin required for the second stage. In the second stage, the Collection plane hits are filled within the drift time window. This refines the hit positioning, resolves overlapping hits, and allows the reconstruction of the individual hit energy deposits [3].

\subsection{Event clusterization}

The aim of the clusterization is to have a rough aggregation of hits in an event. The clusterization results in a rough identification of regions when tracks are separated and of regions when instead the track density is high, like in the case of showers, either electromagnetic or hadronic. 2D clusterization is a prerequisite to $3 \mathrm{D}$ reconstruction, which should reveal the chronological evolution of the particle paths and interactions in the event.

The clusterization is based on hits and it is divided into two steps. First, track-like clusters are identified by taking into account angular distribution of all hits in a view. Second, showerlike clusters are identified, which are built on the basis of track-like clusters. A region with a high density of short track-like clusters is recognized as a shower hence, in this case, all tracklike clusters are transformed into a single shower-like cluster.

\subsection{D event reconstruction}

The real particle track $T$ is observed, in the detector, as a set of three $2 \mathrm{D}$ projections $\mathrm{P}_{\mathrm{II}}(\mathrm{T})$, $\mathrm{P}_{\mathrm{I} 2}(\mathrm{~T}), \mathrm{P}_{\mathrm{C}}(\mathrm{T})$ on to Induction1, Induction2, and Collection wire planes, respectively. In practice, these projections consist of $2 \mathrm{D}$ hits. The 3D fit trajectory $F$ may be projected to the wire planes according to the same operators $\mathrm{P}(F)$. We build the fit trajectory $F$ by minimizing a measure of the distance $D$ between the fit projections and the track hits in all wire planes simultaneously, with constraints $\mathrm{C}_{\mathrm{j}}(F)$, which may include factors such as trajectory curvature and distance to the already identified and reconstructed interaction vertices. This may be expressed with an objective function $\mathrm{G}(F)$ :

$$
\mathrm{G}(F)=\sum_{i} \alpha_{i} \mathrm{D}\left[\mathrm{P}_{i}(T), \mathrm{P}_{i}(F)\right]+\sum_{j} \beta_{j} \mathrm{C}_{j}(F),
$$

where wire planes, denoted with the index $i$, and constraint factors, denoted with the index $j$, have a weighted impact on the overall $\mathrm{G}(F)$ value according to the $\alpha_{\mathrm{i}}$ and $\beta_{\mathrm{j}}$ coefficients. For the practical implementation of constructing the best fit track we have adopted the Polygonal Line Algorithm, PLA [4]. The 3D reconstruction method is deeply discussed in [3]. 


\subsection{Calibration of stopping/decaying particles}

The complete spatial and calorimetrical reconstruction procedure was applied to tracks of stopping particles selected from data collected with the CNGS beam. They were used to compare the theoretical Bethe-Bloch curves describing $\mathrm{d} E / \mathrm{d} x$ evolution along the stopping particle track with the $d E / d x$ sequence reconstructed for data tracks. At the same time the performed analysis was a test of the Birk's low application to the energy correction due to the recombination effect [5]. The particle identification was based on the $\mathrm{d} E / \mathrm{d} x$ trend versus the particle range, which is computed from the point where the particle stops (residual range). The particle identification was performed in order to distinguish tracks compatible with the proton hypothesis from the $\mu / \pi$ hypothesis.

Results, in comparison with the theoretical curves, are shown in figure 2. The theoretical stopping power curves of Bethe-Bloch have been calculated taking into account LA'r properties, shell corrections, and the density effect. Delta rays produced by particles in the examined momentum range have energy low enough to be undistinguishable from the particle track; therefore no delta rays energy restriction has been applied [3].
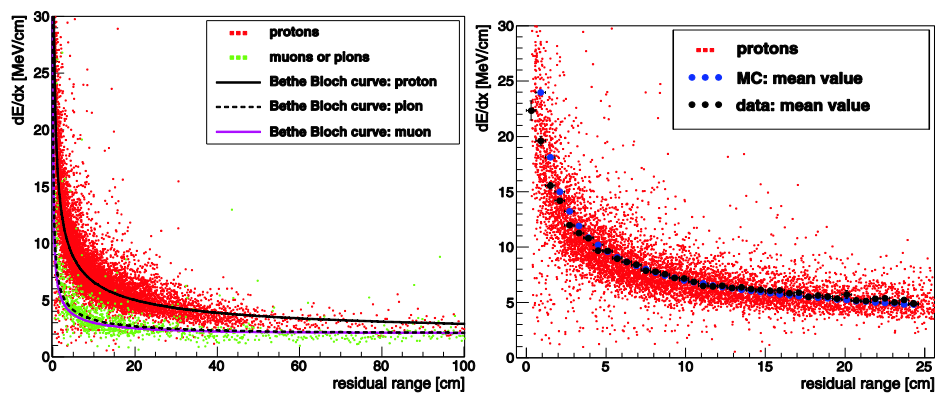

Fig. 2. On the left: the selected sample of data tracks identified as proton or $\mu \pi$ tracks; on the right: $d E / d x$ distributions in $6 \mathrm{~mm}$ bins of residual range: black - data tracks identified as protons (mean value); blue - simulated proton tracks (mean value); points in the background - data tracks identified as protons.

\section{References}

[1] C. Rubbia, The Liquid-argon time projection chamber: a new concept for Neutrino Detector, CERN-EP/77-08.

[2] ICARUS Collaboration, Design, construction and tests of the ICARUS T600 detector, Nucl. Inst. Meth. A556 2006 149-157.

[3] ICARUS Collaboration, Precise 3D Track Reconstruction Algorithm for the ICARUS T600 Liquid Argon Time Projection Chamber Detector, AHEP 2013 (260820).

[4] B. Kegl, A. Krzyżak, T. Linder, and K. Zeger, Learning and design of principal curves, IEEE Transactions on Pattern Analysis and Machine Intelligence 223 2000 281-297.

[5] ICARUS Collaboration, Analysis of the liquid argon purity in the ICARUS T600 TPC, Nucl. Instr. Meth. A516 12004 68-79. 